\title{
Estresse e o estilo de vida dos acadêmicos ingressantes em um curso de graduação em Odontologia
}

Tânia Adas Saliba Rovida*; Doris Hissako Sumida*; Amanda da Silva Santos**; Suzely Adas Saliba Moimaz***; Cléa Adas Saliba Garbin***

Professora Doutora do Programa de Pós-Graduação em Odontologia Preventiva e Social da Faculdade de Odontologia de Araçatuba - UNESP

** Mestranda do Programa de Pós-Graduação em Odontologia Preventiva e Social da Faculdade de Odontologia de Araçatuba - UNESP

*** Professora Titular do Programa de Pós-graduação em Odontologia Preventiva e Social da Faculdade de Odontologia de Araçatuba - UNESP

\section{RESUMO}

O estresse entre os graduandos de cursos da área da saúde tem sido alvo de estudos nos últimos anos. Dentre as mudanças ocorridas no ingresso na universidade, está a adoção de um novo estilo de vida, o qual pode relacionar-se com o estado de ansiedade. $\mathrm{O}$ objetivo nesse estudo foi verificar a autopercepção de estresse, ansiedade e depressão dos ingressantes em um curso de graduação em odontologia, associar à resposta fisiológica do estresse e analisar seu estilo de vida. A amostra foi composta por 25 estudantes de uma universidade pública paulista, ingressantes no curso de odontologia em 2014. Foram aplicados dois instrumentos validados: DASS 21 (Depression, Anxiety and Stress Scale) e PEVI (Perfil do Estilo de Vida Individual), e a dosagem do hormônio cortisol em 75 amostras de saliva, coletadas em três momentos em um dia, utilizando o método laboratorial de ELISA. O estresse e a ansiedade foram presentes em $60 \%$ e a depressão observada em 36\% dos indivíduos.
Quanto ao estilo de vida, $40 \%$ apresentaram perfil pouco desejável e $26 \%$ indesejável no domínio da nutrição, $40 \%$ indesejável na atividade física e nenhum estudante apresentou perfil desejável no controle do estresse. Altas concentrações de cortisol estiveram presentes em $60 \%$ da amostra, porém não houve associação significativa com a percepção do estresse $(\mathrm{p}=0.40)$. Conclui-se que grande parte dos estudantes teve percepção de estresse e ansiedade e altas concentrações de cortisol salivar. A maioria não apresentou padrão de estilo de vida desejável nos domínios de nutrição, atividade física e controle do estresse.

Descritores: Estudantes. Estilo de vida. Estresse. Cortisol.

\section{INTRODUÇÃO}

$\mathrm{O}$ ingresso na vida acadêmica vem acompanhado de inúmeras mudanças no estilo de vida dos estudantes. Alguns fatores como 
morar longe dos pais, aglomerar-se com estranhos, a formação de novos grupos sociais, pressões acadêmicas intensas a busca do equilíbrio dos compromissos sociais com as responsabilidades da vida universitária, uso de bebidas alcoólicas, tabagismo, entre outros, são considerados como as principais causas do estresse entre os ingressantes ${ }^{1-5}$.

Caracterizado a partir de uma perspectiva multidimensional, o estresse é definido de várias formas, tais como uma resposta fisiológica e psicológica, um estímulo, e um processo transacional ${ }^{6-8}$. Em termos gerais o estresse pode ser definido como o esforço de uma função para além da sua capacidade adaptativa ou de tolerância, isto é, o nível que o equilíbrio homeostático consegue atingir sem induzir uma disfunção desadaptativa $^{6}$. Os fatores que agem como agentes estressores podem ser de natureza física, psicológica, emocional e/ou social ${ }^{9,10}$. A maneira como o indivíduo avalia a situação de estresse está relacionada com alguns agentes moderadores, tais como a personalidade, o apoio social, nível educacional, entre outros. Quando o agente estressor atinge uma intensidade que ultrapassa a capacidade do organismo de manter a homeostase, surgem então as doenças consequentes do estresse ${ }^{6}$.

Fisiologicamente, diante de um estímulo estressante, o sistema neuroendócrino, mais especificamente a segunda via, que compreende o hipotálamo, a hipófise e o córtex suprarrenal, libera um dos corticóides mais importantes, o cortisol. Sua liberação é essencial na adaptação ao estresse, porém quando o mesmo ultrapassa os níveis basais pode gerar redução da sensibilidade auditiva, tátil e visual, além de gerar sentimentos relacionados à depressão, ansiedade e descontrole ${ }^{11}$.
Vários estudos mostram o aumento de psicopatologias entre os graduandos da área da saúde, especialmente os ingressantes ${ }^{12,13}$. Estudos ainda revelam que existe uma relação entre o estresse, os hábitos e o estilo de vida, que podem interferir na percepção da condição estressora contribuir para o surgimento de sintomas de ansiedade $\mathrm{e}$ depressão. Durante a formação acadêmica, estima-se que 15 a $25 \%$ dos estudantes universitários apresentem algum transtorno psíquico, principalmente transtornos de ansiedade ${ }^{13,14}$.

O objetivo deste estudo foi verificar a autopercepção de estresse, ansiedade e depressão dos ingressantes de um curso de graduação em odontologia, associar à resposta fisiológica do estresse e analisar seu estilo de vida.

\section{METODOLOGIA}

Trata-se de um estudo observacional de caráter transversal, realizado com os alunos ingressantes no curso de Odontologia ao final do $1^{\circ}$ ano. Foram aplicados dois instrumentos validados, DASS 21 (Depression, Anxiety and Stress Scale) ${ }^{15}$ para a análise da autopercepção dos sinais e sintomas de depressão, ansiedade e estresse, e o PEVI (Perfil do Estilo de Vida Individual) ${ }^{16}$ para a analisar o perfil do estilo de vida. O DASS 21 é um questionário com tradução validada para o português, composto por três subescalas tipo Likert, de quatro pontos. Cada subescala consiste em 7 itens característicos das três manifestações em estudo: depressão, ansiedade e estresse. Por meio deste instrumento, a depressão é caracterizada por baixa afetividade positiva, reduzida autoestima e encorajamento e desespero, ao passo que os sinais de hiperestimulação fisiológica caracterizarão a ansiedade e o estresse será 
detectado pela tensão persistente, irritabilidade e ao baixo limiar de ficar perturbado ou frustrado. Já o PEVI contém 15 questões, divididas em cinco domínios, que são: nutrição, atividade física, comportamento preventivo, relacionamento social e controle do estresse. Este instrumento é interpretado por meio de uma escala de 0 a 9 , classificadas da seguinte forma: de 0 a 1 indesejável, de 2 a 3 pouco desejável, de 4 a 5 moderadamente desejável, de 6 a 7 próximo do desejável e de 8 a 9 desejável/ideal.

Para medir os níveis fisiológicos do estresse, foi realizada a dosagem das concentrações do hormônio cortisol em três amostras de saliva, coletadas em três períodos de um mesmo dia: das 8 às $9 \mathrm{~h}$ da manhã, das 16 às 17 horas da tarde e das 22 às 23 horas da noite. A coleta foi realizada diretamente no tubo Salivette ${ }^{\circledR}$ (Sarstedt, Nümbrecht, Alemanha), sendo o estudante orientado quanto aos procedimentos de coleta: não ingerir alimentos ou escovar os dentes pelo menos uma hora antes da coleta da saliva. Após a coleta, os tubetes foram armazenados no freezer para manter a estabilidade da amostra a $-20^{\circ} \mathrm{C}$. Após o descongelamento e a centrifugação a $1000 \mathrm{xg}$ por 2 minutos, a concentração do cortisol foi detectada por meio do método de ELISA ${ }^{17}$.

A pesquisa foi realizada no final do ano letivo, longe do período de provas, o qual poderia gerar alguma alteração em relação às variáveis estudadas. $\mathrm{O}$ critério de exclusão da amostra foi o uso de medicamentos antidepressivos, pois os mesmos poderiam gerar alterações no padrão de resposta do eixo hipotálamo-pituitária-adrenal e, consequentemente, no nível de cortisol salivar.

A tabulação e análise dos dados foi realizada com os programas Epi Info 7 e Statistica Release 7. Neste estudo foram considerados todos os critérios éticos relacionados à pesquisa envolvendo seres humanos e o projeto foi aprovado por Comitê de Ética institucional (CAAE 44058915. 5.0000.5420).

\section{RESULTADOS}

Participaram do estudo 25 estudantes, cujo perfil em relação ao gênero foi de (6) $28 \%$ do sexo masculino e (19) $72 \%$ do sexo feminino. A faixa etária mostrou-se homogênea, com a média de idade de 19,88 $\pm 2,4$ anos. A Tabela 1 revela as frequências dos sintomas de estresse, ansiedade e depressão, obtidas através do questionário DASS 21. Do total, $60 \%$ relatou alguma manifestação dos sintomas de estresse. Em relação à ansiedade, de forma semelhante ao encontrado para o estresse, foi relatada por $60 \%$ dos alunos. Houve presença de sintomas de depressão em $36 \%$ da amostra, sendo $24 \%$ com grau severo, $8 \%$ moderado e $4 \%$ brando.

$O$ perfil de estilo de vida encontrado entre os jovens mostrou que o domínio da nutrição possui um perfil indesejável e pouco desejável em $64 \%$. Apenas $12 \%$ dos alunos incluem frutas e verduras regularmente em suas refeições, $32 \%$ tem o hábito de evitar alimentos gordurosos e $64 \%$ fazem menos de quatro refeições variadas ao dia, incluindo o café da manhã completo.

Esse padrão mostrou-se mais frequente entre os jovens do sexo masculino (83\%), porém $52 \%$ das jovens também apresentaram esse perfil, indicando que grande parte dos jovens universitários não tem um perfil alimentar ideal.

No domínio da atividade física, $44 \%$ têm comportamento indesejável e $8 \%$ pouco desejável. Atividades físicas moderadas/ intensas e de forma contínua ou acumulada não correspondem à rotina de $64 \%$ dos 
estudantes. Atividades mais leves, como caminhada, ou uso de escadas também não mostraram boa frequência, com apenas $28 \%$ apresentando essa prática. O perfil indesejável no domínio da atividade física esteve presente em $50 \%$ dos rapazes e $42 \%$ das moças. O comportamento preventivo mostrou um perfil próximo do desejável em 56\% dos estudantes, porém apenas $16 \%$ têm conhecimento da sua pressão arterial e dos níveis de colesterol. Além disso, um pouco mais da metade afirmou não fumar ou ingerir álcool. O perfil de comportamento preventivo próximo do desejável e ideal/desejável foi mais comum entre as moças (73\%) do que entre os rapazes $(49 \%)$.

Em relação ao relacionamento social, foi mostrado que grande parte dos estudantes apresentou bom perfil neste domínio, o qual esteve entre ideal e moderadamente desejável. O domínio do controle do estresse, mostrou um perfil indesejável em $16 \%$ dos indivíduos, apresentando-se semelhante entre ambos os sexos. Apenas $32 \%$ apresentou-se próximo do desejável, como observado na Tabela 2 e no Quadro 1.

Tabela 1 - Níveis de depressão, ansiedade e estresse dos alunos ingressantes no curso de Odontologia.

\begin{tabular}{c|cccccc} 
& \multicolumn{2}{|c}{ Estresse } & \multicolumn{2}{c}{ Ansiedade } & \multicolumn{2}{c}{ Depressão } \\
& $\mathrm{n}$ & $\%$ & $\mathrm{n}$ & $\%$ & $\mathrm{n}$ & $\%$ \\
\hline Extra Severo & 5 & 20 & 4 & 16 & 0 & 0 \\
Severo & 3 & 12 & 4 & 16 & 6 & 24 \\
Moderado & 2 & 8 & 4 & 16 & 2 & 8 \\
Brando & 5 & 20 & 3 & 12 & 1 & 4 \\
Normal & 10 & 40 & 10 & 40 & 16 & 64 \\
Total & $\mathbf{2 5}$ & $\mathbf{1 0 0}$ & $\mathbf{2 5}$ & $\mathbf{1 0 0}$ & $\mathbf{2 5}$ & $\mathbf{1 0 0}$ \\
\hline
\end{tabular}

Tabela 2 - Perfil do estilo de vida adotado pelos alunos ingressantes no curso de odontologia.

\begin{tabular}{|c|c|c|c|c|c|c|c|c|c|c|}
\hline & \multicolumn{2}{|c|}{ Nutrição } & \multicolumn{2}{|c|}{$\begin{array}{l}\text { Atividade } \\
\text { Física }\end{array}$} & \multicolumn{2}{|c|}{$\begin{array}{c}\text { Comportamento } \\
\text { Preventivo }\end{array}$} & \multicolumn{2}{|c|}{$\begin{array}{c}\text { Relacionamento } \\
\text { Social }\end{array}$} & \multicolumn{2}{|c|}{$\begin{array}{l}\text { Controle } \\
\text { do Estresse }\end{array}$} \\
\hline & $\mathbf{n}$ & $\%$ & $\mathbf{n}$ & $\%$ & $\mathbf{n}$ & $\%$ & $\mathbf{n}$ & $\%$ & $\mathbf{n}$ & $\%$ \\
\hline Indesejável & 7 & 28 & 11 & 44 & 0 & 0 & 0 & 0 & 4 & 16 \\
\hline Pouco desejável & 8 & 32 & 2 & 8 & 2 & 8 & 1 & 4 & 5 & 20 \\
\hline $\begin{array}{r}\text { Moderadamente } \\
\text { desejável }\end{array}$ & 8 & 32 & 7 & 28 & 6 & 24 & 9 & 36 & 8 & 32 \\
\hline $\begin{array}{r}\text { Próximo do } \\
\text { desejável }\end{array}$ & 1 & 4 & 4 & 16 & 14 & 56 & 8 & 32 & 8 & 32 \\
\hline Ideal/desejável & 1 & 4 & 1 & 4 & 3 & 12 & 7 & 28 & 0 & 0 \\
\hline Total & 25 & 100 & 25 & 100 & 25 & 100 & 25 & 100 & 25 & 100 \\
\hline
\end{tabular}

Os níveis de cortisol apresentados foram classificados em alto, médio e baixo de acordo com os níveis basais de $25 \mathrm{nmol} / \mathrm{L}$.
Concentrações altas de cortisol salivar foram presentes em 60\% dos alunos, com as seguintes médias: no período da manhã 
$31,9 \mathrm{nmol} / \mathrm{L}$, à tarde $26,2 \mathrm{nmol} / \mathrm{L}$ e à noite $23,8 \mathrm{nmol} / \mathrm{L}$, porém não houve associação entre a percepção de estresse e os altos níveis de cortisol $(\mathrm{p}=0.40)$ (Tabela 3$)$, nem com a ansiedade $(\mathrm{p}=0.67)$ e depressão $(\mathrm{p}=0.60)$, considerando a significância de 5\%.

Quadro 1 - Domínios do Perfil do Estilo de Vida dos alunos ingressantes no curso de Odontologia.

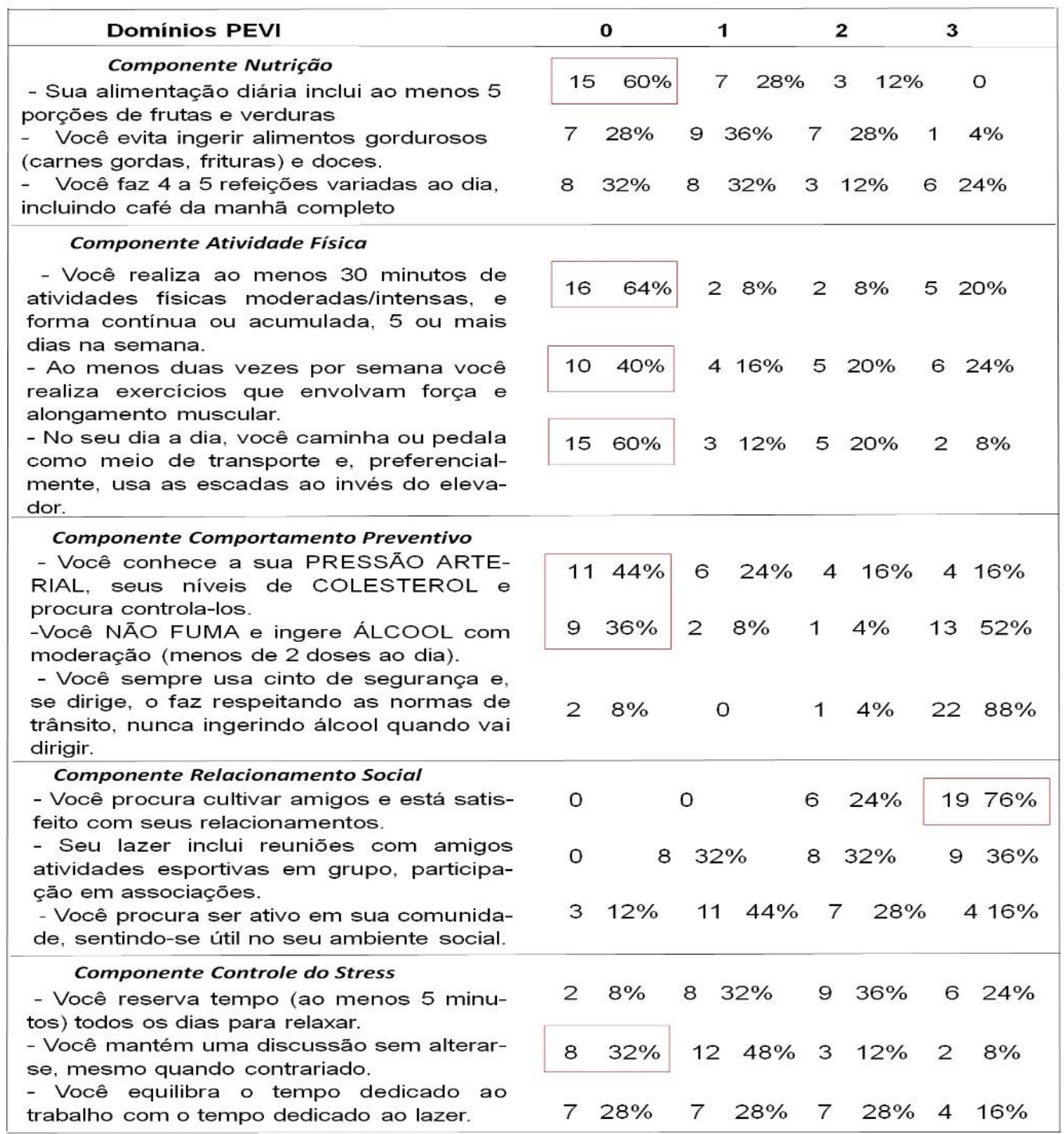

Tabela 3 - Associação entre níveis de cortisol e percepção de estresse dos estudantes.

\begin{tabular}{rrrrrrc}
\multicolumn{7}{c}{ Estresse } \\
Concentração de cortisol & \multicolumn{7}{c}{ Percebido } & Não percebido & Total \\
& $\mathbf{n}$ & $\mathbf{\%}$ & $\mathbf{n}$ & $\mathbf{\%}$ & $\mathbf{n}$ \\
Alta & 9 & 36 & 6 & 24 & 15 \\
Baixa & 6 & 24 & 4 & 16 & 10 \\
Total & $\mathbf{1 5}$ & $\mathbf{6 0}$ & $\mathbf{1 0}$ & $\mathbf{4 0}$ & $\mathbf{2 5}$ \\
\hline
\end{tabular}

$\mathrm{p}=0,40$ 


\section{DISCUSSÃO}

Neste estudo sobre estresse e estilo de vida de estudantes universitários, o perfil dos participantes encontrou-se em concordância com o encontrado em estudos atuais sobre o perfil dos ingressantes em graduação na área da saúde em universidades públicas, com a predominância do sexo feminino e a faixa etária menor ou igual a 21 anos $^{18}$. O perfil do estilo de vida do estudante que ingressa no ensino superior está relacionado à sua capacidade de adaptação a esta nova fase da vida como também foi observado por Costa e Leal ${ }^{19}$. Esta é considerada uma variável diretamente ligada às manifestações de estresse ${ }^{20}$. Neste estudo, todos os perfis encontrados mostraram comportamentos que podem contribuir como fatores estressores e, como consequência, causar o aparecimento de psicopatologias como a ansiedade e a depressão ${ }^{21}$, as quais são significativamente presentes entre graduandos da área da saúde ${ }^{22}$. Quando o indivíduo passa por situações de estresse, o organismo busca $o$ equilíbrio homeostático e, quando este não é atingido, surgem as doenças ${ }^{6,9}$. Um achado importante neste estudo foi a presença dos sintomas de ansiedade na mesma proporção do estresse e a presença de sinais e sintomas de depressão. O instrumento DASS-21, ao investigar a ansiedade, considera a presença de sintomas físicos, ou seja, ela é caracterizada pela hiperestimulação fisiológica, mostrando que em muitos casos houve a resposta física ao estresse na amostra estudada. $\mathrm{O}$ relato de sintomas de depressão entre os alunos, caracterizado por baixa afetividade positiva, reduzida autoestima e encorajamento, assim como desespero, atingiu $36 \%$ dos estudantes. Porém é importante considerar que o relato dos alunos por meio do instrumento DASS-21 refere-se simplesmente a sinais e sintomas dessas manifestações.
Instrumentos de autopercepção são bastante utilizados para este tipo de avaliação. $\mathrm{O}$ estresse percebido também tem sido relacionado com a inteligência emocional (IE) (otimismo/regulação do humor, utilização das emoções, apreciação das emoções e habilidades sociais). Quanto menor for a IE, maior é a percepção de estresse ${ }^{12}$. Portanto, fatores como relacionamento social e controle do estresse analisados no presente estudo podem servir como indicadores da percepção do estresse. Entre os estudantes, $46 \%$ dos que possuíam alguma manifestação de estresse relataram relacionamento social pouco desejável ou moderadamente desejável.

Alguns estudos relacionam a presença de depressão a variáveis como o apetite diminuído e à preocupação com a saúde. Não se alimentar bem representa um fator de risco para o desenvolvimento de doenças crônicodegenerativas e para o comprometimento do bem-estar do indivíduo ${ }^{4,14,23}$. A preocupação com a saúde foi representada pelos domínios do comportamento preventivo e atividade física, que igualmente não apresentaram perfil desejável. Muitos estudantes não conheciam a sua pressão arterial e seus níveis glicêmicos. Além disso, o fumo, o álcool e o sedentarismo estiveram bastante presentes. Ao estudar sobre os hábitos alimentares e níveis de atividade física dos graduandos, este perfil também foi observado por alguns pesquisadores ${ }^{24,25}$. Estes comportamentos, comuns entre os universitários, podem atuar como fatores estressores fisiológicos, mas também podem ser considerados como consequência do estresse psicológico.

Os níveis de cortisol salivar encontrados revelam a presença de estresse fisiológico, ou seja, a resposta fisiológica está presente, porém não houve associação da mesma com a percepção de estresse dos estudantes, a razão 
para isso pode estar relacionada ao tamanho da amostra. Os hábitos saudáveis, especialmente a prática de exercícios físicos, podem diminuir os níveis de cortisol dos estudantes ${ }^{26}$. Daí a importância de políticas que possam incentivar a adoção de hábitos saudáveis entre os universitários.

O tamanho da amostra é uma limitação do estudo, porém, ainda assim, algumas variáveis mostraram-se bastante relevantes e merecem investigações mais aprofundadas, como os padrões alimentares e a presença do estresse como consequência dos problemas enfrentados pelos ingressantes no ensino superior. $\mathrm{O}$ estudo da influência do ambiente acadêmico na adaptação dos estudantes e o aprofundamento das reais causas do estresse também podem contribuir para uma melhor qualidade de vida e integração do acadêmico, e consequentemente, a diminuição de reprovações e desistências ${ }^{27-30}$.

\section{CONCLUSÃO}

Conclui-se que grande parte dos estudantes teve percepção de estresse e ansiedade e altas concentrações de cortisol salivar. A maioria não apresentou padrão de estilo de vida desejável nos domínios de nutrição, atividade física e controle do estresse.

\section{REFERÊNCIAS}

1. Eisenberg D, Gollust SE, Golberstein E, Hefner JL. Prevalence and correlates of depression, anxiety, and suicidality among university students. Am J Orthopsychiatry 2007;77(4):534-42. Disponível em: http:// www.ncbi.nlm.nih.gov/pubmed/18194033

2. Zawawi JA, Hamaideh SH. Depressive symptoms and their correlates with locus of control and satisfaction with life among Jordanian college students. Eur J Psychol 2009;4:71-103. Disponível em:_http://ejop. psychopen.eu/article/view/241
3. Lee RB, Sta. Maria M, Estanislao S, Rodriguez C. Factors associated with depressive symptoms among Filipino university students. PLoS One 2013;8(11):e79825 Disponível em: http://www.ncbi.nlm.nih.gov/pmc/articles/PM C3819248/

4. Brito BJQ, Gordia AP, Quadros TMB. Literature review on the lifestyle of college students. Rev Bras Qual Vida 2014;6(2):66-76. Disponível em: https:// periodicos.utfpr. edu.br/rbqv/article/view/1838

5. Langoski JE, Klipan LB, Souza JÁ, Ferracioli UM, Fadel CB, Bordin D. Influência da trajetória acadêmica sobre o estresse e a percepção de estudantes de Odontologia. Rev Odontol UNESP 2014;43 Spec No:253. Disponível em: http://www.revodontol unesp.com.br/article/5464f18e5ce02a7450000 $\underline{006}$

6. Selye H. The stress of life. New York: McGraw Hill; 1984.

7. Holmes T, Rahe R. The social readjustement rating scale. J Psychosom Res 1967;11(2):21321. Disponível em: http://www.acc.com/ aboutacc/newsroom/pressreleases/upload/SRR $\underline{\text { S.pdf }}$

8. Lazarus R. From psycological stress to emotions: a history of changing outlooks. Annl Rev Psycol 1993; 44:1-21. Disponível em: http://www.annualreviews.org/doi/pdf/10.114 6/annurev.ps.44.020193.000245

9. Lipp MEN. Emotional stress: contribution from internal and external stressors. Rev Psiquiatr Clín 2001;28(6):347-9. http://www. hcnet.usp.br/ipq/revista/vol28/n6/artigos/art34 7.htm

10. Sapolsky RM, Romero LM, Munck AU. How do glucocorticoids influence stress responses? Integrating permissive, suppressive, stimulatory, and preparative actions. Endocr Rev 2000;21(1):55-89. Disponível em: http:// press.endocrine.org/doi/pdf/10.1210/edrv.21.1 .0389

11. Soares AJA, Alves MGP. Cortisol como variável em psicologia da saúde. Psicol Saúde Doenças 2006;7(2):165-77. Disponível em: 
http://www.redalyc.org/articulo.oa?id=362702 $\underline{02}$

12. Pau AKH, Croucher R. Emotional intelligence and perceived stress in dental undergraduates. J Dent Educ 2003;67(9):1023-8. Disponível em: http://www.jdentaled.org/content/67/9/ 1023.long

13. Cavestro JM, Rocha FL. Prevalência de depressão entre estudantes universitários. J Bras Psiquiatr 2006;55(4):264-7. Disponível em: $\quad$ http://www.scielo.br/pdf/jbpsiq/v55n4/ a01v55n4.pdf

14. Sakae TM, Padão DL, Jornada LK. Sintomas depressivos em estudantes da área da saúde em uma Universidade no Sul de Santa Catarina UNISUL. Rev AMRIGS 2010;54(1):38-43. Disponível em: http://www.amrigs.com. br/revista/54-01/11473_sintomas_depressivos. pdf

15. Lovibond P, Lovibond S. The structure of negative emotional states: comparison of the depression anxiety stress scales (DASS) with the beck depression and anxiety inventories. Behav Res Ther 1995;33(3):335-43. Disponível em: http://www.sciencedirect. com/science/article/pii/000579679400075U

16. Nahas MV, Barros MV, Francala V. O pentáculo do bem-estar: base conceitual para avaliação do estilo de vida de indivíduos e grupos. Rev Bras Ativ Fís Saúde. 2000;5(2):48-59. Disponível em: http://periodicos.ufpel.edu.br/ojs2/index.php/ RBAFS/article/view/1002

17. Lima DP, Diniz DG, Moimaz SAS, Sumida DH, Okamoto AC. Saliva: reflection of the body. Int J Infect Dis. 2010;14(3):e184-8. Disponível em: http://www.sciencedirect.com /science/article/pii/S1201971209002033

18. Nardelli GG, Gaudenci EM, Garcia BB, Carleto CT, Gontijo LM, Pedrosa LAK. Perfil dos alunos ingressantes dos cursos da área da saúde de uma universidade federal. Rev Enferm Atenção Saúde 2013;2(1):3-12. Disponível em: http://www.uftm.edu.br/ revistaeletronica/index.php/enfer/article/view/ $\underline{405}$
19. Costa ES, Leal I. Um olhar sobre a saúde psicológica dos estudantes do ensino superior: avaliar para intervir. In: Actas do 7th Congresso Nacional de Psicologia da Saúde; 2008; Porto, Portugal. p. 214. Disponível em: http://www.isabel-leal.com/Portals/1/PDFs/7 congresso/vii-congresso-saude-pp-213216.pdf

20. Uraz A, Tocak YS, Yozgatligil C, Cetiner S, Bal B. Psychological well-being, health, and stress sources in Turkish dental students. J Dent Educ 2013;77(10):1345-55. Disponível em: http://www.jdentaled.org/content/77/10/ 1345.long

21. Marcondelli P, Costa THM, Schmitz BAS. Nível de atividade física e hábitos alimentares de universitários do $3^{\circ}$ ao $5^{\circ}$ semestres da área da saúde. Rev Nutr 2008;21(1):39-47. Disponível em: http://www.scielo.br/pdf/rn/ v21n1/a05v21n1.pdf

22. Costa SC, Diefenbach AL, Diefenbach M, Santos RN. Avaliacão do nível de estresse através do teste de inventário de sintomas de estresse de lipp (issl) em acadêmicos da área de saúde Manaus-AM. Rev Amazônica 2010;4(1):7-18. Disponível em: http://dialnet. unirioja.es/servlet/articulo?codigo $=4028854$

23. Paixão LA, Dias RMR, Prado WL. Estilo de vida e estado nutricional de universitários ingressantes em cursos da área de saúde do Recife/PE. Rev Bras Ativ Fis Saúde. 2010;15(3):145-50. Disponível em: http://www.periodicos.ufpel.edu.br/ojs2/index .php/RBAFS/article/viewFile/714/720

24. Soar C, Silva OS, Lira JG. Consumo alimentar e atividade física de estudantes universitários da área de saúde. Rev Univap 2012;18(31):417. Disponível em: http://revista.univap.br/ index.php/revistaunivap/article/view/58

25. Santos JJA, Saracini N, Silva WC, Guilherme JH, Costa TA, Silva MRAG. Estilo de vida relacionado à saúde de estudantes universitários: comparação entre ingressantes e concluintes ABCS Health Sci 2014;39(1):1723. Disponível em: http://www.portalnepas. org.br/abcshs/article/view/256

26. Camacho E, Vega-Michel C, Orejudo S. 
Niveles de cortisol y estilo de vida en estiudiantes universitarios sanos de México y España. Rev Latinoam Med Conductual 2011;1(2):29-40. Disponível em: http://www. journals.unam.mx/index.php/rlmc/article/view 124880

27. Cavalcanti AL, Lima WG, Marques JLS, Alves HFC, Granville-Garcia AF. Motivos de ingresso e de evasão dos acadêmicos de Odontologia de uma instituição pública. Rev Odontol UNESP 2010;39(2):95-9. Disponível em: http://revodontolunesp.com.br/files/v39 n2/v39n2a05.pdf

28. Machado FC, Souto DMA, Freitas CHSM, Forte FDS. Odontologia como escolha: perfil de graduandos e perspectiva para o futuro profissional. Rev ABENO 2010;10(2):27-34 Disponível em: http://revabeno.emnuvens. com.br/revabeno/article/view/17/17

29. Pereira GM, Arantes DCB, Giovannini JFBG, Amorim JNC, Mendonça SMS. Avaliação diagnóstica: uma ferramenta para avaliar a evolução do desempenho dos alunos do Curso de Odontologia do Centro Universitário Newton Paiva. Ver ABENO 2012;12(2)142-6. Disponível em: http://revabeno.emnuvens. com.br/revabeno/article/view/116/107

30. Santos BRM, Gonzales OS, Carrer FCA, Araújo ME. Perfil e expectativas dos ingressantes da Faculdade de Odontologia da USP: uma visão integrada com as diretrizes curriculares nacionais e o sistema único de saúde. Rev ABENO 2015;15(1):28-37. Disponível em: http://revabeno.emnuvens. com.br/revabeno/article/view/150/134

\section{ABSTRACT \\ Stress and lifestyle of freshmen in dentistry course}

The stress among undergraduate students in health care courses has been investigated in recent years. Among the changes on these students when starting their courses at the university, it was noticed the adopting of a new lifestyle, which may be related to the state of anxiety. The aim of this study was to verify the self-perception of stress, anxiety and depression of freshmen in undergraduate dental students, associating physiological response to stress and analyzing their lifestyles. The study was based on laboratory samples from 25 students that started their dentistry course in 2014 in a public institution. Two validated instruments were applied: DASS 21 (Depression, Anxiety and Stress Scale) and PEVI (Individual Lifestyle Profile) and the dosage of the hormone cortisol in 75 saliva samples collected at three times on a day, using the ELISA laboratory method. The stress and anxiety were present in $60 \%$ and depression was observed in $36 \%$ of the subjects. As for lifestyle, $40 \%$ had less than desirable profile and $26 \%$ undesirable in the field of nutrition, $40 \%$ undesirable physical activity and none had desired profile in stress management. High cortisol concentrations were present in $60 \%$ of the sample, but there was no significant association with the perception of stress $(\mathrm{p}=$ $0.40)$. It was concluded that most of the students had perceived stress, anxiety, and high concentrations of salivary cortisol. Most did not present desirable lifestyle patterns in the areas of nutrition, physical activity and stress management.

Descriptors: Students. Lifestyle. Stress. Cortisol.

Correspondência para:

Profa. Dra. Tânia Adas Saliba Rovida

e-mail: tasaliba@ foa.unesp.br

Programa de Pós-Graduação em Odontologia

Preventiva e Social

Rua José Bonifácio, 1193.

Vila Mendonça 16015-050 - Araçatuba/SP 